\title{
Head-to-Head Comparison of Clinical Scores for Predicting Stroke Associated Pneumonia after Intracerebral Hemorrhage
}

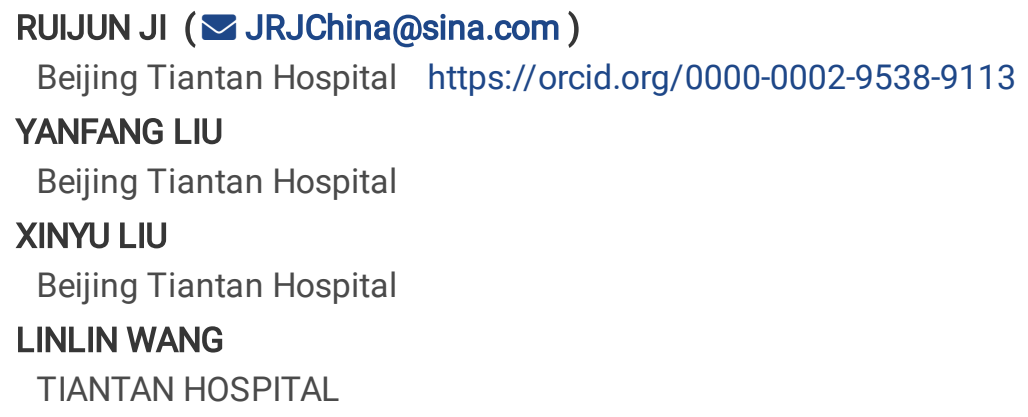

YANFANG LIU

Beijing Tiantan Hospital

XINYU LIU

Beijing Tiantan Hospital

LINLIN WANG

TIANTAN HOSPITAL

\section{DANDAN WANG}

Beijing Tiantan Hospital

WENJUAN WANG

Beijing Tiantan Hospital

RUNHUA ZHANG

Beijing Tiantan Hospital

RUIXUAN JIANG

Beijing Tiantan Hospital

JIAOKUN JIA

Beijing Tiantan Hospital

HAO FENG

TIANTAN HOSPITAL

ZEYU DING

TIANTAN HOSPITAL

GAIFEN LIU

Beijing Tiantan Hospital

JINGJING LU

Beijing Tiantan Hospital

YIJU

Beijing Tiantan Hospital

XINGQUAN ZHAO

Beijing Tiantan Hospital

\section{Research}

Keywords: Intracerebral hemorrhage, Stroke associated pneumonia, risk model, discrimination, calibration, comparison

Posted Date: December 11th, 2020

DOI: https://doi.org/10.21203/rs.3.rs-125417/v1

License: (c) (i) This work is licensed under a Creative Commons Attribution 4.0 International License. Read Full License 


\section{Abstract}

Background: Despite advances in medical knowledge, treatment for ICH remains strictly supportive with not many evidencebased interventions currently available. Stroke associated pneumonia (SAP) is a common medical complication after stroke and has significant impact on stroke outcomes. In the study, we aimed to systematically compare discrimination and calibration of clinical scores with regard to in-hospital SAP after ICH.

Methods: The validation cohort was derived from the Beijing Registration of Intracerebral Hemorrhage. The SAP was diagnosed according to the criteria for hospital-acquired pneumonia of Center for Disease Control and Prevention. Five clinical scores were included in the study: the ICH-APS-A, ICH-ASP-B, ISAN, ACCD4 and PASS score. Discrimination was assessed by calculating the area under the receiver operating characteristic curve (AUROC). Pairwise AUROC was compared by using Delong's method. Calibration was assessed by performing the Hosmer-Lemeshow goodness-of-fit test and plot of observed versus predicted risk according to 10 deciles of the predicted risk. The Cox and Snell R-square and Nagelkerke R-square of the Hosmer-Lemeshow goodness-of-fit test were calculated.

Results: A total number of 1964 patients were enrolled. The mean age was $56.8 \pm 14.4$ and $67.6 \%$ were male. The median admission NIHSS was 11 (IQR: 3-21). The median length of stay (LOS) was 16 days (IQR: 8-22). A total number of 575 (29.2\%) patients was diagnosed with in-hospital SAP after ICH. The AUROC of five clinical scores ranged from 0.732 to 0.800 . In pairwise comparison, the ICH-APS-B $(0.800,95 \% \mathrm{Cl}=0.780-0.820, \mathrm{P}<0.001)$ showed statistically better discrimination than other risk models (all $\mathrm{P}<0.001$ ). All clinical scores performed better among patients with LOS longer than 72 hours. The ICH-APS-B (0.827, $95 \% \mathrm{Cl}=0.806-0.848, \mathrm{P}<0.001)$ still showed statistically better discrimination than other risk models in patients with LOS longer than 72 hours (all P<0.001). The ICH-SAP-B had the largest Cox and Snell R-square of the Hosmer-Lemeshow test for in-hospital SAP after ICH.

Conclusion: Several models are externally validated to be effective for risk stratification and outcome prediction for in-hospital SAP after ICH, which would be useful tools for personalized care and clinical trials in prevention of SAP after ICH.

\section{Background}

Spontaneous intracerebral hemorrhage (ICH) accounts for approximately $15-20 \%$ of all strokes and is one of leading causes of mortality and morbidity worldwide[1, 2]. Despite advances in medical knowledge, treatment for ICH remains strictly supportive with not many evidence-based interventions currently available[3, 4].

Stroke associated pneumonia (SAP) is a common medical complication after stroke and has significant impact on stroke outcomes. Evidence showed that SAP not only increase the length of hospital stay and medical cost, but also is an important risk factor of mortality and morbidity after stroke[5]. In addition, it was found that pneumonia could increase risk of several nonpneumatic medical complications, such as deep vein thrombosis, gastrointestinal bleeding and atrial fibrillation[6]. Meanwhile, previous study showed that SAP was more common in patients with $\mathrm{ICH}$ than those with acute ischemic stroke (AIS) [6, 7]. These data point out the need for more aggressive SAP prophylaxis among patients with $\mathrm{ICH}$.

Several clinical scores have been developed for predicting SAP after ICH, such as the VHA score[8], ICH-APS-A[9], ICH-ASP-B[9], ISAN[10], ACCD4[11] and PASS[12]. The ISAN[10], ACCD4[11] and PASS score[12] included ICH patients in the derivation cohorts, while the ICH-APS-A[9] and ICH-APS-B[9] scores were developed exclusively for ICH. Although some of these ICH risk models have been internally or externally validated, none of them has been universally accepted and consistently used in routine clinical practice and clinical research. In addition, with many grading system available, it is becoming increasingly difficult for clinician and researcher to determine which risk models provide optimal predictability and reliability in clinical practice and clinical trials. Therefore, it is necessary to conduct head-to-head comparison of these models in an independent cohort.

In the study, we aimed to systematically compare discrimination and calibration of clinical scores with regard to in-hospital SAP after ICH following the TRIPOD (transparent reporting of a multivariable prediction model for individual prognosis or diagnosis) guideline[13, 14]. 


\section{Methods}

\section{Validation cohort}

The validation cohort was derived from the Beijing Registration of Intracerebral Hemorrhage, which was a multicenter, prospective and observational cohort study. A total number of thirteen hospitals in Beijing area participated in the study. To be eligible for the study, subjects had to meet the following criteria: (1) age 18 years or older; (2) hospitalized with a primary diagnosis of spontaneous ICH and confirmed by brain CT or MRI; (3) direct admission to hospital from a physician's clinic or emergency department; (4) written informed consent from patients or their legal representatives. The study protocol was approved by the Institutional Review Board (IRB) of the Beijing Tiantan Hospital (KY2014-023-02).

\section{Data Collection And Definition Of Variables}

A standardized electronic case report form (eCRF) was used for data collection. Participating centers collected data and submitted it online to the coordinating center at Beijing Tiantan Hospital. For this study, the following candidate variables were analyzed: (1) demographics; (2) time from onset to hospital (hours); (3) stroke risk factors: hypertension, diabetes mellitus, dyslipidemia, atrial fibrillation, history of stroke/TIA, myocardial infarction, heart failure, current smoking and alcohol consumption; (4) pre-stroke modified Rankin Scale (mRS) score; (5) pre-admission antithrombotic medications; (6) admission stroke severity based on the National Institutes of Health Stroke Scale score (NIHSS) and the Glasgow Coma Scale (GCS) score; (7) admission blood pressure ( $\mathrm{mmHg}$ ): (8) admission laboratory tests; (9) neuroimaging variables: hematoma volume (measured using the $A B C / 2$ method[15]), hematoma location (supratentorial or infratentorial ICH), intraventricular extension (presence or absence) and subarachnoid extension (presence or absence). (10) etiology diagnosis (primary or secondary ICH); (11) surgical treatment (craniotomy evacuation, minimal-invasive surgical therapy or brain ventricle puncture and drainage); (12) withdrawal of medical care; and (13) length of hospital stay (LOS).

\section{Diagnosis Of Sap}

In-hospital SAP was diagnosed by treating physician according to the criteria for hospital-acquired pneumonia of Center for Disease Control and Prevention[16, 17], on a basis of clinical and laboratory indices of respiratory tract infection (fever, cough, new purulent sputum, auscultatory respiratory crackles) and supported by typical chest X-ray findings. In the study, pneumonia occurred before stroke was not considered.

\section{Statistical analysis}

Categorical variables were summarized as proportions. Continuous variables were summarized with mean and standard deviation (SD) or median and interquartile range (IQR). Chi-square or Fisher exact test was used to compare categorical variables and Mann-Whitney test or independent t-test was employed to compare continuous variables between groups.

By systematic searching, six clinical scores were identified, which could be used to predict SAP after ICH. The VHA score[8] cannot be validated in the study due to that we did not have information on "Found-down at symptom onset". Finally, five clinical scores were included in the study: the ICH-APS-A[9], ICH-ASP-B[9], ISAN[10], ACCD 4 ${ }^{[11]}$ and PASS[12]. Discrimination was assessed by calculating the area under the receiver operating characteristic curve (AUROC)[13, 14]. Pairwise AUROC was compared by using Delong's method[18]. Sensitivity, specificity, positive predict value (PPV), and negative predictive value (NPV) were calculated at each risk models' maximum Youden Index. Calibration was assessed by performing the Hosmer-Lemeshow goodness-of-fit test and plot of observed versus predicted risk according to 10 deciles of the predicted risk. The Cox and Snell Rsquare and Nagelkerke R-square of the Hosmer-Lemeshow goodness-of-fit test were calculated[13, 14]. Due to that the HosmerLemeshow test has been shown to be overly sensitive[19], Pearson's correlation coefficient between observed and predicted risk was calculated as well. 
All tests were 2-tailed and statistical significance was determined at a level of 0.05 . Statistical analysis was performed using SAS 9.1 (SAS Institute, Cary, NC), SPSS 26.0 (SPSS Inc., Chicago, IL), and Medcalc software 12.3 (MedCalc).

\section{Results}

\section{Patient characteristics}

From December 2014 to September 2016, a total number of 1964 patients were enrolled in the Beijing Registration of Intracerebral Hemorrhage. The clinical characteristics are shown in Table 1. The mean age was $56.8 \pm 14.4$ and $67.6 \%$ were male. The median time from onset to hospital was 4.0 hours (IQR: 1.90-11.1). The median NIHSS and GCS score on admission was 11 (IQR: 3-21) and 14 (IQR: 8-15), respectively. The median hematoma volume on CT was $15.8 \mathrm{~cm}^{3}$ (IQR: 6.0-38.6). The median length of hospital stay was 16 days (IQR: 8-22). A total number of 575 (29.2\%) patients was diagnosed with in-hospital SAP after ICH. Compared to patients without in-hospital SAP, those with in-hospital SAP after ICH were older, with higher proportion of dysphagia, dysarthria, hematoma intraventricular extension, subarachnoid extension, receiving surgical treatment and higher NIHSS score, blood pressure, blood glucose, hematoma volume and longer hospital stay (Table 1). 
Table 1

baseline characteristics

\begin{tabular}{|c|c|c|c|c|}
\hline & $\begin{array}{l}\text { Overall } \\
(n=1964)\end{array}$ & $\begin{array}{l}\text { SAP } \\
(n=1389)\end{array}$ & $\begin{array}{l}\text { without SPA } \\
(n=575)\end{array}$ & $P$ value \\
\hline \multicolumn{5}{|l|}{ Demographics } \\
\hline Age, y, median (IQR) & $56.8 \pm 14.4$ & $55.9 \pm 14.1$ & $59.0 \pm 14.6$ & $<0.001$ \\
\hline Gender (male), n (\%) & $1327(67.6)$ & $925(66.6)$ & $402(69.9)$ & 0.16 \\
\hline Onset to hospital (hours), median (IQR) & $4.0(1.90-11.0)$ & $4.13(1.98-12.8)$ & $3.45(1.82-8.00)$ & 0.001 \\
\hline \multicolumn{5}{|l|}{ Risk factors, $\mathrm{n}(\%)$} \\
\hline Hypertension & $1367(69.6)$ & $955(68.8)$ & $412(71.7)$ & 0.22 \\
\hline Diabetes mellitus & $289(14.7)$ & $205(14.8)$ & $84(14.6)$ & 1.00 \\
\hline Dyslipidemia & $184(9.4)$ & $138(9.9)$ & $46(8.0)$ & 0.20 \\
\hline Atrial fibrillation & $30(1.5)$ & $19(1.4)$ & $11(1.9)$ & 0.42 \\
\hline History of stroke/TIA & $309(15.7)$ & $199(14.3)$ & $110(19.1)$ & 0.01 \\
\hline Myocardial infarction & $38(1.9)$ & $25(1.8)$ & $13(2.3)$ & 0.47 \\
\hline Heart failure & $8(0.4)$ & $5(0.4)$ & $3(0.5)$ & 0.70 \\
\hline Current smoker & $628(32.0)$ & $421(30.3)$ & $207(36.0)$ & 0.02 \\
\hline Alcohol consumption & $716(36.5)$ & $500(36.0)$ & $216(37.6)$ & 0.53 \\
\hline Pre-admission anticoagulation, $\mathrm{n}(\%)$ & $21(1.1)$ & $13(0.9)$ & $8(1.4)$ & 0.46 \\
\hline Pre-admission antiplatelet, $\mathrm{n}(\%)$ & $277(14.1)$ & $178(12.8)$ & $99(17.2)$ & 0.01 \\
\hline Pre-admission statins, $\mathrm{n}(\%)$ & $113(5.8)$ & $72(5.2)$ & $41(7.1)$ & 0.11 \\
\hline Pre-stroke mRS score, median (IQR) & $0(0-0)$ & $0(0-0)$ & $0(0-0)$ & 0.02 \\
\hline Admission NIHSS score, median (IQR) & $11(3-21)$ & $8(2-15)$ & $20(12-27)$ & $<0.001$ \\
\hline Admission GCS score, median (IQR) & $14(8-15)$ & $14(12-15)$ & $9(6-13)$ & $<0.001$ \\
\hline Admission dysphagia, $\mathrm{n}(\%)$ & $666(33.9)$ & $286(20.6)$ & $380(66.1)$ & $<0.001$ \\
\hline Admission dysarthria, $\mathrm{n}(\%)$ & $918(46.7)$ & $599(43.1)$ & $319(55.5)$ & $<0.001$ \\
\hline Admission SBP (mm Hg), median (IQR) & $165(147-186)$ & $161(145-182)$ & $173(150-191)$ & $<0.001$ \\
\hline Admission DBP (mm Hg), median (IQR) & $96(82-109)$ & $95(81-108)$ & $98(84-110)$ & 0.009 \\
\hline Admission WBC, $10^{9} / \mathrm{L}$, median (IQR) & $9.79(7.35-13.0)$ & $9.14(7.03-11.9)$ & $11.5(8.30-14.7)$ & $<0.001$ \\
\hline Admission glucose (mmol/L), median (IQR) & $5.85(4.89-7.39)$ & $5.54(4.72-6.93)$ & $6.57(5.53-8.14)$ & $<0.001$ \\
\hline Admission creatinine (umol/L), median (IQR) & $63.4(52.7-77.0)$ & $63.1(52.9-76.5)$ & $63.8(52.0-77.1)$ & 0.89 \\
\hline Hematoma location & & & & 0.17 \\
\hline Supratentorial ICH,n (\%) & $1752(89.2)$ & $1248(89.8)$ & $504(87.7)$ & \\
\hline Infratentorial ICH, n (\%) & $212(10.8)$ & $141(10.2)$ & $71(12.3)$ & \\
\hline
\end{tabular}

Abbreviation: SAP, Stroke Associated Pneumonia; IQR, Interquartile Range; NIHSS, National Institutes of Health Stroke Scale score; GCS, Glasgow Coma Scale; SBP, Systolic Blood Pressure; DBP, Diastolic Blood Pressure; WBC, White Cell Count; ICH, Intracerebral Hemorrhage; mRS, modified Rankin Scale. 


\begin{tabular}{|c|c|c|c|c|}
\hline & $\begin{array}{l}\text { Overall } \\
(n=1964)\end{array}$ & $\begin{array}{l}\text { SAP } \\
(n=1389)\end{array}$ & $\begin{array}{l}\text { without SPA } \\
(n=575)\end{array}$ & $P$ value \\
\hline Hematoma volume $\left(\mathrm{cm}^{3}\right)$, median (IQR) & $15.8(6.0-38.6)$ & $12.0(5.0-28.1)$ & $30.0(12.8-59.3)$ & $<0.001$ \\
\hline Intraventricular extension, $\mathrm{n}(\%)$ & $655(33.4)$ & $368(26.5)$ & $287(49.9)$ & $<0.001$ \\
\hline Subarachnoid extension, n (\%) & $264(13.4)$ & $152(10.9)$ & $112(19.9)$ & $<0.001$ \\
\hline Etiology diagnosis, n (\%) & & & & 0.61 \\
\hline Primary ICH & $1785(90.9)$ & $1259(90.6)$ & $526(91.5)$ & \\
\hline Secondary ICH & $159(8.1)$ & $117(8.4)$ & $42(7.3)$ & \\
\hline Primary IVH & $20(1.0)$ & $13(0.9)$ & $7(1.2)$ & \\
\hline Withdrawal of medical care, $\mathrm{n}(\%)$ & $139(7.1)$ & $86(6.2)$ & $53(9.2)$ & 0.02 \\
\hline Surgical treatment, $\mathrm{n}(\%)$ & $366(18.6)$ & $159(11.4)$ & $207(36.0)$ & $<0.001$ \\
\hline length of hospital stay, median (IQR) & $16(8-22)$ & $15(8-20)$ & $19(8-29)$ & $<0.001$ \\
\hline
\end{tabular}

\section{Length Of Stay And In-hospital Sap After Ich}

Patients characteristics stratified by LOS ( $\leq 72$ vs. $>72$ hours) are shown in supplementary table 1 . Compared to patients with LOS longer than 72 hours, those with LOS less than 72 hours had significantly higher admission NIHSS score $(P<0.001)$, lower GCS score $(P<0.001)$ and larger hematoma volume $(P<0.001)$. However, the proportion of in-hospital SAP between two groups was not statistically significant $(30.2 \%$ vs. $29.2 \%, P=0.75)$. It was found that patients with LOS less than 72 hours had significantly higher rate of in-hospital mortality $(40.6 \%$ vs. $7.4 \%, P<0.001)$ and withdraw of medical care $(18.4 \%$ vs. $5.7 \%, P<0.001)$ than those with LOS longer than72 hours (Table 2). 
Table 2

Baseline characteristics stratified by length of stay

\begin{tabular}{|c|c|c|c|c|}
\hline & $\begin{array}{l}\text { Overall } \\
(n=1964)\end{array}$ & $\begin{array}{l}\text { LOS } \leq 72 h \\
(n=212)\end{array}$ & $\begin{array}{l}\text { LOS }>72 h \\
(n=1752)\end{array}$ & $P$ value \\
\hline \multicolumn{5}{|l|}{ Demographics } \\
\hline Age, y, median (IQR) & $56.8 \pm 14.4$ & $57.1 \pm 13.3$ & $56.7 \pm 14.4$ & 0.73 \\
\hline Gender (male), n (\%) & $1327(67.6)$ & $145(68.4)$ & $1182(67.5)$ & 0.82 \\
\hline Onset to hospital (hours), median (IQR) & $4.0(1.90-11.0)$ & $4.01(1.92-12.5)$ & $3.28(1.98-5.96)$ & $<0.001$ \\
\hline \multicolumn{5}{|l|}{ Risk factors, $\mathrm{n}(\%)$} \\
\hline Hypertension & $1367(69.6)$ & $152(71.7)$ & $1215(69.3)$ & 0.53 \\
\hline Diabetes mellitus & $289(14.7)$ & $34(16.0)$ & $255(14.6)$ & 0.53 \\
\hline Dyslipidemia & $184(9.4)$ & $22(10.4)$ & $162(9.2)$ & 0.62 \\
\hline Atrial fibrillation & $30(1.5)$ & $4(1.9)$ & $26(1.5)$ & 0.41 \\
\hline History of stroke/TIA & $309(15.7)$ & $27(12.7)$ & $282(16.1)$ & 0.23 \\
\hline Myocardial infarction & $38(1.9)$ & $5(2.4)$ & $33(1.9)$ & 0.60 \\
\hline Heart failure & $8(0.4)$ & $2(0.9)$ & $6(0.3)$ & 0.21 \\
\hline Current smoker & $628(32.0)$ & $60(28.3)$ & $568(32.4)$ & 0.24 \\
\hline Alcohol consumption & $716(36.5)$ & $70(33.0)$ & $646(36.9)$ & 0.29 \\
\hline Pre-admission anticoagulation, $\mathrm{n}(\%)$ & $21(1.1)$ & $2(0.9)$ & $19(1.1)$ & 1.00 \\
\hline Pre-admission antiplatelet, $\mathrm{n}(\%)$ & $277(14.1)$ & $29(13.7)$ & $248(14.2)$ & 0.92 \\
\hline Pre-stroke mRS score, median (IQR) & $0(0-0)$ & $0(0-0)$ & $0(0-0)$ & 0.38 \\
\hline Admission NIHSS score, median (IQR) & $11(3-21)$ & $26(12-33)$ & $10(3-18)$ & $<0.001$ \\
\hline Admission GCS score, median (IQR) & $14(8-15)$ & $6(3-13)$ & $14(10-15)$ & $<0.001$ \\
\hline Admission dysphagia, $\mathrm{n}(\%)$ & $666(33.9)$ & $104(49.1)$ & $562(32.1)$ & $<0.001$ \\
\hline Admission dysarthria, $\mathrm{n}(\%)$ & $918(46.7)$ & $128(60.4)$ & $790(45.1)$ & $<0.001$ \\
\hline Admission SBP (mm Hg), median (IQR) & $165(147-186)$ & $173(150-200)$ & $164(146-185)$ & $<0.001$ \\
\hline Admission DBP (mm Hg), median (IQR) & $96(82-109)$ & $97(85-110)$ & $95(82-102)$ & 0.15 \\
\hline Admission WBC, $10^{9} / \mathrm{L}$, median (IQR) & $9.79(7.35-13.0)$ & $12.4(9.31-17.3)$ & $9.49(7.20-12.4)$ & $<0.001$ \\
\hline Admission glucose (mmol/L), median (IQR) & $5.85(4.89-7.39)$ & $8.84(6.84-11.6)$ & $7.18(6.02-8.88)$ & $<0.001$ \\
\hline Admission creatinine (umol/L), median (IQR) & $63.4(52.7-77.0)$ & $64.9(53.6-83.0)$ & $63.1(52.6-76.2)$ & 0.17 \\
\hline Hematoma location & & & & $<0.001$ \\
\hline Supratentorial ICH,n (\%) & $1752(89.2)$ & $172(81.1)$ & $1580(90.2)$ & \\
\hline Infratentorial $\mathrm{ICH}, \mathrm{n}(\%)$ & $212(10.8)$ & 40 (18.9) & $172(9.8)$ & \\
\hline Hematoma volume $\left(\mathrm{cm}^{3}\right)$, median (IQR) & $15.8(6.0-38.6)$ & $40.9(12.0-78.1)$ & $15.0(5.8-34.8)$ & $<0.001$ \\
\hline
\end{tabular}

Abbreviation: IQR, Interquartile Range; NIHSS, National Institutes of Health Stroke Scale score; GCS, Glasgow Coma Scale; SBP, Systolic Blood Pressure; DBP, Diastolic Blood Pressure; WBC, White Cell Count; ICH, Intracerebral Hemorrhage; mRS, modified Rankin Scale. 


\begin{tabular}{|c|c|c|c|c|}
\hline & $\begin{array}{l}\text { Overall } \\
(n=1964)\end{array}$ & $\begin{array}{l}L O S \leq 72 h \\
(n=212)\end{array}$ & $\begin{array}{l}\text { LOS }>72 h \\
(n=1752)\end{array}$ & $P$ value \\
\hline Intraventricular extension, $\mathrm{n}(\%)$ & $655(33.4)$ & $79(37.3)$ & $576(32.9)$ & 0.21 \\
\hline Subarachnoid extension, n (\%) & $264(13.4)$ & $55(25.9)$ & 209(11.9) & $<0.001$ \\
\hline Etiology diagnosis, n (\%) & & & & 0.02 \\
\hline Primary ICH & 1785(90.9) & $204(96.2)$ & $1581(90.2)$ & \\
\hline Secondary ICH & $159(8.1)$ & $7(3.3)$ & $152(8.7)$ & \\
\hline Primary IVH & $20(1.0)$ & $1(0.5)$ & $19(1.1)$ & \\
\hline Withdrawal of medical care, $\mathrm{n}(\%)$ & $139(7.1)$ & $39(18.4)$ & $100(5.7)$ & $<0.001$ \\
\hline Surgical treatment, $\mathrm{n}(\%)$ & $366(18.6)$ & $18(8.5)$ & $348(19.9)$ & $<0.001$ \\
\hline in-hospital SAP, n (\%) & $575(29.3)$ & $64(30.2)$ & $511(29.2)$ & 0.75 \\
\hline in-hospital mortality, n (\%) & $216(11.0)$ & $86(40.6)$ & $130(7.4)$ & $<0.001$ \\
\hline
\end{tabular}

\section{Comparison Of Model Discrimination For In-hospital Sap}

Figure 1 shows discrimination of five clinical scores with regard to in-hospital SAP after ICH. The AUROC of 6 clinical scores ranged from 0.732 to 0.800 (Table 3). The sensitivity, specificity, PPV, NPV and maximum Youden Index for predicting in-hospital SAP after ICH are shown in Table 2. The ICH-SAP-B showed the maximum Youden Index. In pairwise comparison, the ICH-APS-B $(0.800,95 \% \mathrm{Cl}=0.780-0.820, \mathrm{P}<0.001)$ showed statistically better discrimination than other risk models for in-hospital SAP after $\mathrm{ICH}($ all $\mathrm{P}<0.001)$. 
Table 3

Discrimination of risk models with regard to in-hospital SAP after ICH

\begin{tabular}{|c|c|c|c|c|c|c|c|c|c|c|}
\hline & AUROC & $95 \% \mathrm{Cl}$ & $\stackrel{\Delta}{\text { AUROC* }}$ & $\begin{array}{l}\text { P } \\
\text { value }^{\&}\end{array}$ & $\begin{array}{l}\text { Youden } \\
\text { Index }\end{array}$ & Cutoff & Sensitivity & Specificity & PPV & NPV \\
\hline \multicolumn{11}{|l|}{$\begin{array}{l}\text { Overall } \\
\text { cohort } \\
(\mathrm{N}= \\
1964)\end{array}$} \\
\hline $\begin{array}{l}\text { ICH-APS- } \\
\text { B (2014) }\end{array}$ & 0.800 & $\begin{array}{l}0.780- \\
0.820\end{array}$ & Reference & $\ldots$ & 0.495 & 6 & 0.777 & 0.718 & 0.533 & 0.886 \\
\hline $\begin{array}{l}\text { ICH-APS- } \\
\text { A (2014) }\end{array}$ & 0.787 & $\begin{array}{l}0.766- \\
0.808\end{array}$ & 0.013 & $\begin{array}{l}< \\
0.001\end{array}$ & 0.462 & 6 & 0.724 & 0.739 & 0.534 & 0.866 \\
\hline $\begin{array}{l}\text { ISAN } \\
(2015)\end{array}$ & 0.755 & $\begin{array}{l}0.732- \\
0.777\end{array}$ & 0.045 & $\begin{array}{l}< \\
0.001\end{array}$ & 0.421 & 8 & 0.683 & 0.737 & 0.519 & 0.849 \\
\hline $\begin{array}{l}\text { ACDD }^{4} \\
(2017)\end{array}$ & 0.755 & $\begin{array}{l}0.735- \\
0.774\end{array}$ & 0.045 & $<.001$ & 0.456 & 3 & 0.662 & 0.794 & 0.571 & 0.850 \\
\hline $\begin{array}{l}\text { PASS } \\
(2018)\end{array}$ & 0.732 & $\begin{array}{l}0.708- \\
0.756\end{array}$ & 0.068 & $\begin{array}{l}< \\
0.001\end{array}$ & 0.388 & 6 & 0.692 & 0.696 & 0.485 & 0.845 \\
\hline \multicolumn{11}{|l|}{$\begin{array}{l}\text { LOS } \leq 72 \\
\text { hours }(\mathrm{N} \\
=288)\end{array}$} \\
\hline $\begin{array}{l}\text { ICH-APS- } \\
\text { B (2014) }\end{array}$ & 0.639 & $\begin{array}{l}0.574- \\
0.703\end{array}$ & Reference & $\ldots$ & 0.265 & 6 & 0.830 & 0.435 & 0.392 & 0.853 \\
\hline $\begin{array}{l}\text { ICH-APS- } \\
\text { A (2014) }\end{array}$ & 0.637 & $\begin{array}{l}0.573- \\
0.702\end{array}$ & 0.002 & 0.92 & 0.272 & 6 & 0.773 & 0.500 & 0.405 & 0.833 \\
\hline $\begin{array}{l}\text { ISAN } \\
(2015)\end{array}$ & 0.599 & $\begin{array}{l}0.533- \\
0.665\end{array}$ & 0.040 & 0.09 & 0.214 & 9 & 0.784 & 0.430 & 0.377 & 0.819 \\
\hline $\begin{array}{l}\text { ACDD }^{4} \\
(2017)\end{array}$ & 0.648 & $\begin{array}{l}0.580- \\
0.716\end{array}$ & 0.009 & 0.73 & 0.319 & 2 & 0.704 & 0.615 & 0.446 & 0.826 \\
\hline $\begin{array}{l}\text { PASS } \\
(2018)\end{array}$ & 0.627 & $\begin{array}{l}0.556- \\
0.697\end{array}$ & 0.012 & 0,70 & 0.249 & 7 & 0.534 & 0.715 & 0.452 & 0.777 \\
\hline \multicolumn{11}{|l|}{$\begin{array}{l}\text { LOS }>72 \\
\text { hours }(N \\
=1676)\end{array}$} \\
\hline $\begin{array}{l}\text { ICH-APS- } \\
\text { B (2014) }\end{array}$ & 0.827 & 0.806.-0.848 & Reference & $\ldots$ & 0.533 & 6 & 0.768 & 0.765 & 0.573 & 0.890 \\
\hline $\begin{array}{l}\text { ICH-APS- } \\
\text { A (2014) }\end{array}$ & 0.811 & $\begin{array}{l}0.789- \\
0.832\end{array}$ & 0.016 & $\begin{array}{l}< \\
0.001\end{array}$ & 0.493 & 6 & 0.714 & 0.779 & 0.570 & 0.869 \\
\hline $\begin{array}{l}\text { ISAN } \\
(2015)\end{array}$ & 0.780 & $\begin{array}{l}0.756- \\
0.804\end{array}$ & 0.047 & $<001$ & 0.455 & 8 & 0.661 & 0.793 & 0.568 & 0.851 \\
\hline $\begin{array}{l}\text { ACDD }^{4} \\
(2017)\end{array}$ & 0.773 & $\begin{array}{l}0.747- \\
0.798\end{array}$ & 0.055 & $<.001$ & 0.482 & 1 & 0.679 & 0.802 & 0.585 & 0.859 \\
\hline $\begin{array}{l}\text { PASS } \\
\text { (2018) }\end{array}$ & 0.750 & $\begin{array}{l}0.724- \\
0.775\end{array}$ & 0.077 & $\begin{array}{l}< \\
0.001\end{array}$ & 0.421 & 6 & 0.702 & 0.718 & 0.505 & 0.855 \\
\hline \multicolumn{11}{|c|}{$\begin{array}{l}{ }^{\star} \triangle \mathrm{AUROC} \text { denotes the difference in AUROC between the ICH-SAP-B and compared scores with regard to SAP after ICH. \& P } \\
\text { value of comparing paired AUROC with Delong's method. }\end{array}$} \\
\hline $\begin{array}{l}\text { obreviati } \\
\text { terval; P }\end{array}$ & $\begin{array}{l}\mathrm{CH}, \text { IntI } \\
\text { Oositive }\end{array}$ & $\begin{array}{l}\text { erebral Hem } \\
\text { edictive Valu }\end{array}$ & age; AUR & rea U & $\begin{array}{l}r \text { the } R \\
\text { Value }\end{array}$ & er Op & ng Chara & istic Curv & Con & \\
\hline
\end{tabular}


All risk models showed much better discrimination in patients with LOS longer than 72 hours (Table 3). Among patients with LOS longer than 72 hours, the ICH-APS-B $(0.827,95 \% \mathrm{Cl}=0.806-0.848, \mathrm{P}<0.001)$ still showed statistically better discrimination than other risk models with regard to in-hospital SAP after ICH (all $\mathrm{P}<0.001$ ).

\section{Comparison Of Model Calibration For In-hospital Sap}

The predicted and observed risk according to 10 deciles of the predicted risk of in-hospital SAP after ICH was plotted (supplementary Fig. 1). The results of Hosmer-Lemeshow test are shown in Table 4. The ICH-APS-B have a significance level of Hosmer-Lemeshow test greater than 0.05 in overall cohort, as indicated that the observed values are not statistically different from the expected values. The ICH-SAP-B had the largest Cox and Snell R-square. Similar results were found for patients with LOS longer than 72 hours. Due to that the Hosmer-Lemeshow test has been shown to be overly sensitive to trivial deviations from the ideal fit, Pearson correlation coefficient between predicted and observed risk was calculated. All correlation coefficient of five models was greater than 0.90 (all P<0.001). The ICH-SAP-B had the largest Pearson correlation coefficient.

Table 4

Calibration of risk models with regard to in-hospital SAP after ICH

\begin{tabular}{|c|c|c|c|}
\hline \multirow[t]{2}{*}{ International ICH models } & \multicolumn{3}{|c|}{ Goodness of fit test with regard to SAP after ICH } \\
\hline & $P$ value & Cox and Snell $\mathrm{R}^{2}$ & Nagelkerke $\mathrm{R}^{2}$ \\
\hline \multicolumn{4}{|l|}{ Overall cohort $(\mathrm{N}=1964)$} \\
\hline ICH-APS-B (2014) & 0.08 & 0.210 & 0.299 \\
\hline ICH-APS-A (2014) & $<0.05$ & 0.194 & 0.277 \\
\hline ISAN (2015) & $<0.05$ & 0.155 & 0.221 \\
\hline $\operatorname{ACDD}^{4}(2017)$ & $<0.05$ & 0.178 & 0.253 \\
\hline PASS (2018) & $<0.05$ & 0.128 & 0.183 \\
\hline \multicolumn{4}{|l|}{ LOS $\leq 72$ hours $(N=288)$} \\
\hline ICH-APS-B (2014) & 0.05 & 0.055 & 0.078 \\
\hline ICH-APS-A (2014) & 0.09 & 0.053 & 0.074 \\
\hline ISAN (2015) & 0.04 & 0.030 & 0.043 \\
\hline $\operatorname{ACDD}^{4}(2017)$ & 0.30 & 0.072 & 0.102 \\
\hline PASS (2018) & 0.37 & 0.036 & 0.050 \\
\hline \multicolumn{4}{|l|}{ LOS $>72$ hours $(N=1676)$} \\
\hline ICH-APS-B (2014) & 0.10 & 0.251 & 0.358 \\
\hline ICH-APS-A (2014) & $<0.05$ & 0.228 & 0.326 \\
\hline ISAN (2015) & $<0.05$ & 0.190 & 0.271 \\
\hline $\operatorname{ACDD}^{4}(2017)$ & 0.09 & 0.202 & 0.288 \\
\hline PASS (2018) & $<0.05$ & 0.149 & 0.212 \\
\hline
\end{tabular}

\section{Discussion}

In the study, we systematically compared discrimination and calibration of five clinical scores with regard to in-hospital SAP after $\mathrm{ICH}$. The AUROC ranged from 0.732 to 0.800 in the overall cohort. In pairwise comparison, the ICH-APS-B showed statistically 
better discrimination than other risk models with regard to in-hospital SAP after ICH. All risk models showed much better discrimination among patients with LOS longer than 72 hours. The ICH-APS-B still showed statistically better discrimination than other risk models for in-hospital SAP after ICH in patients with LOS longer than 72 hours. The ICH-SAP-B had the largest Cox and Snell R-square of the Hosmer-Lemeshow goodness of fit test for predicting in-hospital SAP after ICH.

Previous studies have shown that clinical scores for predicting SAP performed better in sensitivity analyses for patients survival beyond 48-72 hours after ICH[9, 10]. It is interesting to figure out the potential reasons. We compared the baseline characteristics between patients with LOS less than 72 hours and those longer than 72 hours. It was found that patients with shorter LOS had significantly more severe neurological deficit on admission, such as with higher NIHSS score, lower GCS score and larger hematoma volume. However, these patients did not have correspondingly increased risk of in-hospital SAP after ICH. The rates of in-hospital SAP between two groups was not statistically different. Further, we found that patients with shorter LOS had significantly higher proportion of in-hospital mortality and withdraw of medical care (Supplementary Table 1). We conjectured that the contradiction between neurological severity and risk of in-hospital SAP after ICH in patients with LOS less than 72 hours might be due to that patients died or left hospital before pneumonia occurred. This might be potential reason for less sensitivity of these clinical scores for in-hospital pneumonia after ICH in this group of patients.

Despite advances in medical knowledge, treatment for ICH remains strictly supportive with not many evidence-based interventions currently available[3, 4]. Evidence showed that SAP not only increase the length of hospital stay and medical cost, but also is an important risk factor of mortality and morbidity after stroke. In addition, previous study showed that the rate of SAP was higher among patients with ICH than those with AIS[6]. These data point out the need for more aggressive SAP prophylaxis among patients with ICH in comparison with AIS. Several clinical scores have been developed for predicting SAP after ICH. Although some risk models have been internally or externally validated, none of them has been universally accepted and consistently used in routine clinical practice and clinical research. In this large ICH cohort $(n=1964)$, the ICH-APS-B showed statistically better discrimination than other risk models with regard to in-hospital SAP after ICH. Meanwhile, the ICH-APS-B showed the largest Cox and Snell R-square of the Hosmer-Lemeshow goodness of fit test for predicting in-hospital SAP after ICH. Though it is promising, caution need to be taken when interpreting the results: first, the cohorts used to develop these clinical scores are different. The ISAN, ACCD4 and PASS prediction score included ICH patients in the derivation cohorts, while the ICHAPS-A and ICH-APS-B scores were developed exclusively in ICH. Second, the baseline characteristics of study populations for derivation and validation of these clinical scores are different. Finally, there might be complex genetic, social, economic factors as well as regional management philosophies and preferences that are difficult to account for when risk models are developed or applied to a distinct population. These clinical scores need to be further validated in more populations and larger samples.

In two recent large randomized trials (stroke-INF[20] and PASS[21]), preventive antibiotic therapy did not improve functional outcome after stroke. These trials selected patients by symptoms and signs and prevention strategies are developed for the average person (one size fit all), with less consideration for the differences in SAP risk between individuals. In this way, it is inevitable to include patients with unbalanced, too high, or too low risk of developing SAP in these clinical trials. These validated clinical scores could be used to stratify patients for risk of SAP after stroke and then test prophylactic antibiotics in different risk stratifications. Clinical trials conducted in this way will allow us to clarify more accurately prophylactic antibiotics will work in which risk stratification patients. Whether preventive antibiotic therapy in a high-risk subgroup patients could improve functional outcome after ICH warrants to be investigated.

Our study has limitation that deserve mention. First, like all observational studies, we cannot rule out the possibility that additional baseline variable (unmeasured confounders) might have some impact on the development of in-hospital SAP after $\mathrm{ICH}$, such as use of angiotensin converting enzyme inhibitors,[22] acid-suppressive medications[23], oral hygiene[24] and biomarkers for stroke-induced immunodepression[25, 26]. Second, we cannot have all elements required for all risk models. For example, we did not have information about "Found-down at symptom onset" and VHA score[8] cannot be validated in the study. Third, our study included only hospitalized patients and those patients died in emergency room or treated in outpatient clinics were not included. Finally, validation cohorts originated from Asian population and the ICH models needed to be further validated in different populations. 


\section{Conclusion}

Several risk models are externally validated to be effective for risk stratification and outcome prediction with regard to in-hospital SAP after ICH, which would be useful tools for personalized care and clinical trials in prevention of SAP after ICH.

\section{Abbreviations}

Intracerebral hemorrhage (ICH)

Transparent reporting of a multivariable prediction model for individual prognosis or diagnosis (TRIPOD) guideline

modified Rankin Scale (mRS)

Institutional Review Board (IRB)

electronic case report form (eCRF)

National Institutes of Health Stroke Scale score (NIHSS)

Glasgow Coma Scale (GCS)

Length of hospital stay (LOS)

Standard deviation (SD)

Interquartile range (IQR)

Area under the receiver operating characteristic curve (AUROC)

Positive predict value (PPV)

Negative predictive value (NPV)

China National Stroke Registry (CNSR)

\section{Declarations}

1. The study protocol was approved by the Institutional Review Board (IRB) of the Beijing Tiantan Hospital (KY2014-023-02).

2. All authors are consent for publication.

3. Availability of data and materials: the corresponding author has the responsibility on behalf of Beijing Registration of Intracerebral Hemorrhage investigators and has full access to the final database. The datasets used and/or analyzed during the current study are available from the corresponding author on reasonable request

4. Competing interests

Dr. Ruijun Ji reports no disclosures.

Dr. Wenjuan Wang reports no disclosures.

Dr. Xinyu Liu reports no disclosures.

Dr. Linlin Wang reports no disclosures.

Dr. Ruixuan Jiang reports no disclosures.

Dr. Runhua Zhang reports no disclosures. 
Dr. Dandan Wang reports no disclosures.

Dr. Jiaokun Jia reports no disclosures.

Dr. Hao Feng reports no disclosures.

Dr. Zeyu Ding reports no disclosures.

Dr. Yanfang Liu reports no disclosures.

Dr. Gaifen Liu reports no disclosures.

Dr. Jingjing Lu reports no disclosures.

Dr. Yi Ju reports no disclosures.

Dr. Xingquan Zhao reports no disclosures.

5. Funding

This study was sponsored by Capital health research and development of special (2011-2004-03) and Beijing Municipal Science \& Technology Commission (Z131107002213009). This study was partially supported by the Nova Program of Beijing Science and Technology Commission (2008B30), National Natural Science Foundation of China (81471208, 81641162), Beijing high-level healthy human resource project (014-3-033) and Shandong Province Key Innovation Project (2019JZZY020901).

6. Authors' contributions

Conception and design: RJ, XZ.

Acquisition of data: Beijing Registration of Intracerebral Hemorrhage investigators.

Analysis or interpretation of data: All authors.

Drafting the manuscript: $\mathrm{RJ}, \mathrm{XL}, \mathrm{LW}$.

Revising the manuscript for important intellectual content: RJ, XZ, GF, YJ.

Statistical analysis: RZ, GL, RJ.

Obtaining funding: $X Z, R J$.

Supervision: $X Z$.

\section{References}

1. Virani SS, Alonso A, Benjamin EJ, Bittencourt MS, Callaway CW, Carson AP, Chamberlain AM, Chang AR, Cheng S, Delling FN, et al. Heart Disease and Stroke Statistics-2020 Update: A Report From the American Heart Association. Circulation. 2020;141(9):e139-596.

2. Wang W, Jiang B, Sun H, Ru X, Sun D, Wang L, Wang L, Jiang Y, Li Y, Wang Y, et al. Prevalence, Incidence, and Mortality of Stroke in China: Results from a Nationwide Population-Based Survey of 480687 Adults. Circulation. 2017;135(8):759-71.

3. Hemphill JC 3rd, Greenberg SM, Anderson CS, Becker K, Bendok BR, Cushman M, Fung GL, Goldstein JN, Macdonald RL, Mitchell PH, et al. Guidelines for the Management of Spontaneous Intracerebral Hemorrhage: A Guideline for Healthcare Professionals From the American Heart Association/American Stroke Association. Stroke. 2015;46(7):2032-60.

4. Steiner T, Al-Shahi Salman R, Beer R, Christensen H, Cordonnier C, Csiba L, Forsting M, Harnof S, Klijn CJ, Krieger D, et al. European Stroke Organisation (ESO) guidelines for the management of spontaneous intracerebral hemorrhage. Int J Stroke. 
2014;9(7):840-55.

5. Teh WH, Smith CJ, Barlas RS, Wood AD, Bettencourt-Silva JH, Clark AB, Metcalf AK, Bowles KM, Potter JF, Myint PK. Impact of stroke-associated pneumonia on mortality, length of hospitalization, and functional outcome. Acta Neurol Scand. 2018;138(4):293-300.

6. Ji R, Wang D, Shen H, Pan Y, Liu G, Wang P, Wang Y, Li H, Wang Y. China National Stroke Registry I: Interrelationship among common medical complications after acute stroke: pneumonia plays an important role. Stroke. 2013;44(12):3436-44.

7. Kwan J, Pickering RM, Kunkel D, Fitton C, Jenkinson D, Perry VH, Ashburn AM. Stroke Association Rehabilitation Research C: Impact of stroke-associated infection on long-term survival: a cohort study. J Neurol Neurosurg Psychiatry. 2013;84(3):297304.

8. Chumbler NR, Williams LS, Wells CK, Lo AC, Nadeau S, Peixoto AJ, Gorman M, Boice JL, Concato J, Bravata DM. Derivation and validation of a clinical system for predicting pneumonia in acute stroke. Neuroepidemiology. 2010;34(4):193-9.

9. Ji R, Shen H, Pan Y, Du W, Wang P, Liu G, Wang Y, Li H, Zhao X, Wang Y, et al. Risk score to predict hospital-acquired pneumonia after spontaneous intracerebral hemorrhage. Stroke. 2014;45(9):2620-8.

10. Smith CJ, Bray BD, Hoffman A, Meisel A, Heuschmann PU, Wolfe CD, Tyrrell PJ, Rudd AG. Intercollegiate Stroke Working Party G: Can a novel clinical risk score improve pneumonia prediction in acute stroke care? A UK multicenter cohort study. J Am Heart Assoc. 2015;4(1):e001307.

11. Kumar S, Marchina S, Massaro J, Feng W, Lahoti S, Selim M, Herzig SJ. ACDD(4) score: A simple tool for assessing risk of pneumonia after stroke. J Neurol Sci. 2017;372:399-402.

12. Westendorp WF, Vermeij JD, Hilkens NA, Brouwer MC, Algra A, van der Worp HB, Dippel DW, van de Beek D, Nederkoorn PJ. Development and internal validation of a prediction rule for post-stroke infection and post-stroke pneumonia in acute stroke patients. Eur Stroke J. 2018;3(2):136-44.

13. Moons KG, Altman DG, Reitsma JB, loannidis JP, Macaskill P, Steyerberg EW, Vickers AJ, Ransohoff DF, Collins GS. Transparent Reporting of a multivariable prediction model for Individual Prognosis or Diagnosis (TRIPOD): explanation and elaboration. Ann Intern Med. 2015;162(1):W1-73.

14. Collins GS, Reitsma JB, Altman DG, Moons KG. Transparent reporting of a multivariable prediction model for individual prognosis or diagnosis (TRIPOD): the TRIPOD statement. BMJ. 2015;350:g7594.

15. Kothari RU, Brott T, Broderick JP, Barsan WG, Sauerbeck LR, Zuccarello M, Khoury J. The ABCs of measuring intracerebral hemorrhage volumes. Stroke. 1996;27(8):1304-5.

16. Garner JS, Jarvis WR, Emori TG, Horan TC, Hughes JM. CDC definitions for nosocomial infections, 1988. Am J Infect Control. 1988;16(3):128-40.

17. Smith CJ, Kishore AK, Vail A, Chamorro A, Garau J, Hopkins SJ, Di Napoli M, Kalra L, Langhorne P, Montaner J, et al. Diagnosis of Stroke-Associated Pneumonia: Recommendations From the Pneumonia in Stroke Consensus Group. Stroke. 2015;46(8):2335-40.

18. DeLong ER, DeLong DM, Clarke-Pearson DL. Comparing the areas under two or more correlated receiver operating characteristic curves: a nonparametric approach. Biometrics. 1988;44(3):837-45.

19. Kramer AA, Zimmerman JE. Assessing the calibration of mortality benchmarks in critical care: The Hosmer-Lemeshow test revisited. Crit Care Med. 2007;35(9):2052-6.

20. Kalra L, Irshad S, Hodsoll J, Simpson M, Gulliford M, Smithard D, Patel A, Rebollo-Mesa I, Investigators S-I. Prophylactic antibiotics after acute stroke for reducing pneumonia in patients with dysphagia (STROKE-INF): a prospective, clusterrandomised, open-label, masked endpoint, controlled clinical trial. Lancet. 2015;386(10006):1835-44.

21. Westendorp WF, Vermeij JD, Zock E, Hooijenga IJ, Kruyt ND, Bosboom HJ, Kwa VI, Weisfelt M, Remmers MJ, ten Houten R, et al. The Preventive Antibiotics in Stroke Study (PASS): a pragmatic randomised open-label masked endpoint clinical trial. Lancet. 2015;385(9977):1519-26.

22. Caldeira D, Alarcao J, Vaz-Carneiro A, Costa J. Risk of pneumonia associated with use of angiotensin converting enzyme inhibitors and angiotensin receptor blockers: systematic review and meta-analysis. BMJ. 2012;345:e4260. 
23. Marchina S, Doros G, Modak J, Helenius J, Aycock DM, Kumar S. Acid-suppressive medications and risk of pneumonia in acute stroke patients: A systematic review and meta-analysis. J Neurol Sci. 2019;400:122-8.

24. Wagner C, Marchina S, Deveau JA, Frayne C, Sulmonte K, Kumar S. Risk of Stroke-Associated Pneumonia and Oral Hygiene. Cerebrovasc Dis. 2016;41(1-2):35-9.

25. Mengel A, Ulm L, Hotter B, Harms H, Piper SK, Grittner U, Montaner J, Meisel C, Meisel A, Hoffmann S. Biomarkers of immune capacity, infection and inflammation are associated with poor outcome and mortality after stroke - the PREDICT study. BMC Neurol. 2019;19(1):148.

26. Nam KW, Kim TJ, Lee JS, Kwon HM, Lee YS, Ko SB, Yoon BW. High Neutrophil-to-Lymphocyte Ratio Predicts StrokeAssociated Pneumonia. Stroke. 2018;49(8):1886-92.

27. Additional file 1.

28. Title. additional table 1.

29. File. format: PDF.

30. Title of data. Components of clinical scores for predicting stroke-associated pneumonia after ICH.

31. Description of data. supplementary table 1 provided relevant information on components of clinical scores for predicting stroke-associated pneumonia after ICH.

\section{Additional file 2.}

33. Title. additional table 2.

34. File. format: PDF.

35. Title of data. Performance of the clinical scores for predicting SAP in the derivation and internal validation cohorts.

36. Description of data. additional table 2 showed relevant information on Performance of the clinical scores for predicting SAP in the derivation and internal validation cohorts.

\section{Additional file 3.}

38. Title. additional table 3.

39. File. format: PDF.

40. Title of data. Plot of observed versus predicted risk of in-hospital SAP after ICH in the derivation and validation cohorts.

41. Description of data. additional table 3 showed plot of observed versus predicted risk of in-hospital SAP after ICH overall cohorts according to 10 deciles of predicted risk in overall cohort $(\mathrm{N}=1964)$. All correlation coefficient of five models was greater than 0.90 (all $\mathrm{P}<0.001$ ), which indicated excellent calibration.. The ICH-SAP-B had the largest Pearson correlation coefficient $(n=1964 ; r=0.98, P<0.001)$.

\section{Figures}




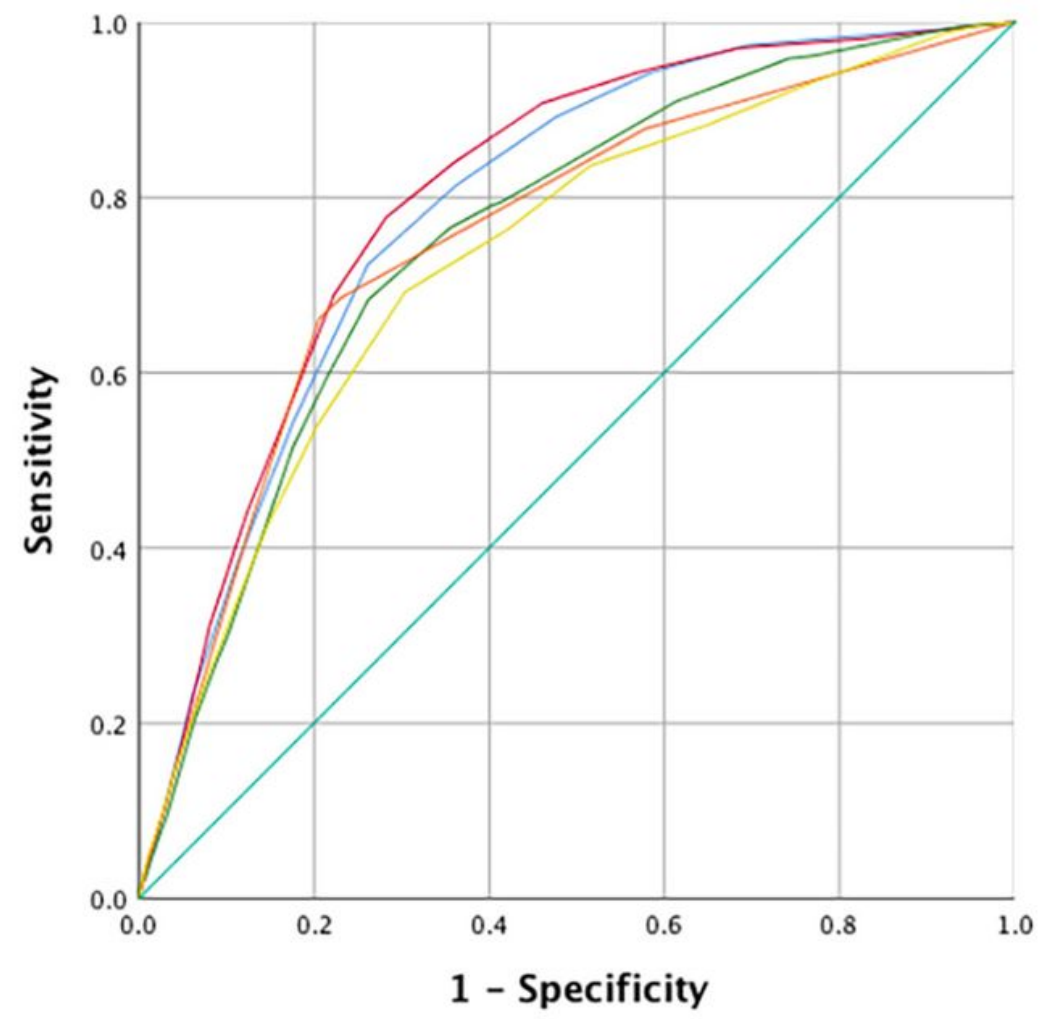

ICH_APS_A
- ICH_APS_B
ISAN
ACDD4
PASS
Reference Line

Figure 1

Predictive performance of clinical scores with regard to in-hospital SAP after ICH $(n=1964)$
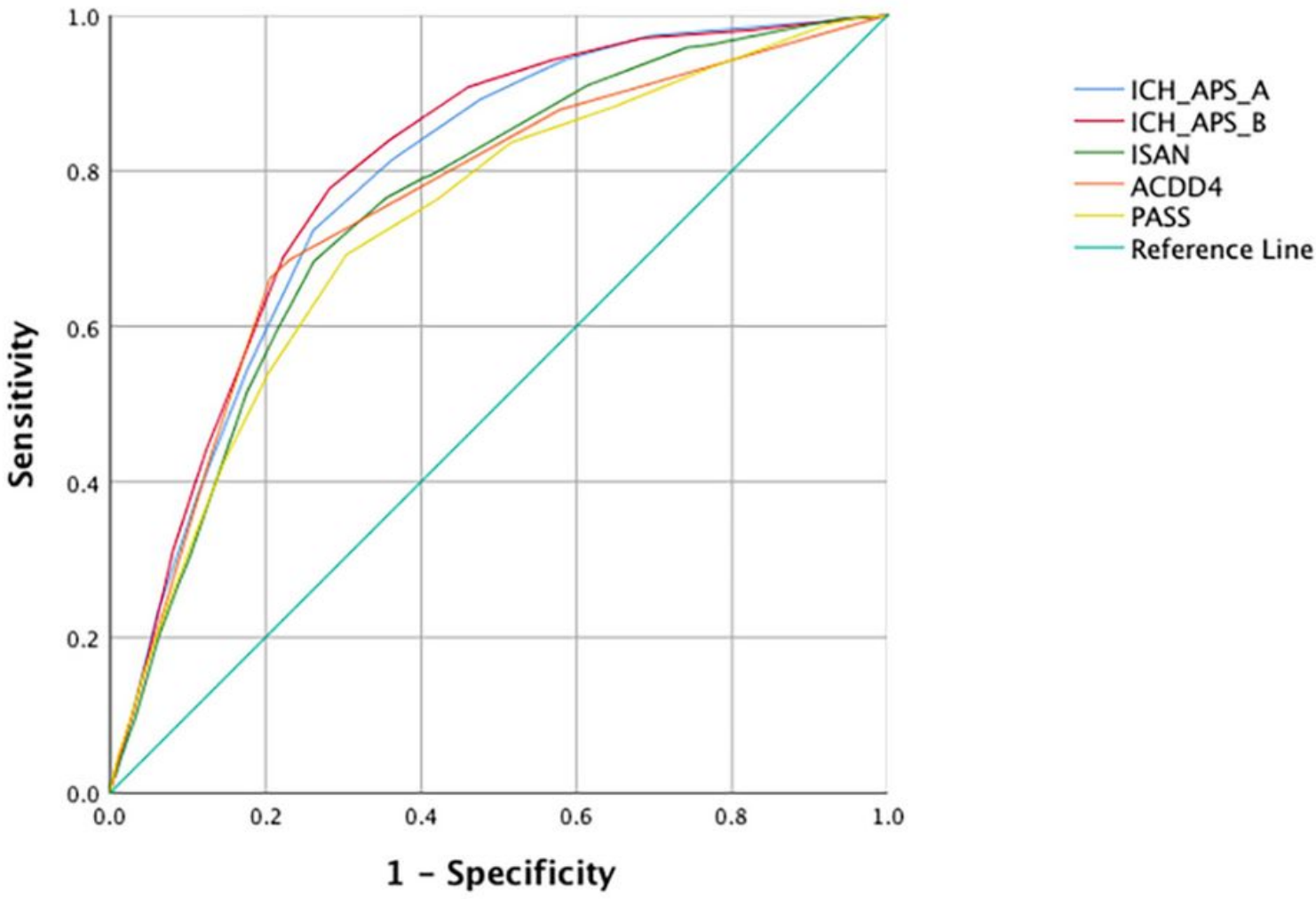


\section{Figure 1}

Predictive performance of clinical scores with regard to in-hospital SAP after ICH ( $n=1964)$

\section{Supplementary Files}

This is a list of supplementary files associated with this preprint. Click to download.

- Supplementarymaterial.docx

- Supplementarymaterial.docx 